\title{
Research on Oscillation-Free Robust Control for Active Joint Dental Automation
}

\author{
Farzin Pilta ${ }^{1}$, Meysam Esmaeili ${ }^{1}$, Mohammad Ali Tayebi ${ }^{1}$, Mahsa Piltan ${ }^{1}$, \\ Mojtaba Yaghoot $^{1}$ and Nasri B. Sulaiman ${ }^{1,2}$ \\ ${ }^{1}$ Intelligent System and Robotic Lab, Iranian Institute of Advance Science and \\ Technology (IRAN SSP), Shiraz/Iran \\ ${ }^{2}$ Department of Electrical Engineering, University Putra Malaysia, Malaysia \\ Email: piltan_f@iranssp.org,WWW.IRANSSP.ORG/english
}

\begin{abstract}
Design a robust oscillation-free controller for multi input-multi output (MIMO) nonlinear uncertain dynamical system (sensitive dental joint) is the main objective in this research. In this paper, robust sliding mode controller will be selected as a main control technique and linear controller will be design to improve the tability and robustness to control of dental joint. The proposed approach effectively combines of design methods from switching sliding mode controller, and linear Propontional-Integral-Derivative (PID) control to improve the performance, stability and robustness of the sliding mode controller.

Conventional sliding mode controller has two important subparts, switching and equivalent. Switching part (discontinugas part) is ve y important in uncertain condition but it causes chattering phenomenon Tosolve the chattering, the most common method used is linear boundary layer saturation method, but this method lost the stability. To reduce the chattering with respect to stabilityand robustness; linear controller is added to the switching part of the sliding mode cantroller. The linear controller is to reduce the role of sliding surface slope and swithing (sign) function. This controller improves the stability and robustness, reduces the chattering as well and reduces the level of energy due to the torque performance as well
\end{abstract}

Keywords: Conventional sliding mode controller, linear controller, chattering phenomenon, stability, robusthess, multi degrees of freedom dental joint

\section{Introduction}

One of the best ways to maintenance of comprehensive oral health is Nanodentistry. It is employing nanomaterials, biotechnology, including tissue engineering and ultimately, dental nanorobotics. Nanodentistry can do oral health maintenance using mechanical dentifrobots, like local anesthesia, dentition renaturalization, permanent hypersensitivity cure, complete orthodontic realignments, covalently bonded diamond dised enamel and ec. Any product containing nano particles are Nano products that can be made by combining atomic elements to create mechanical nanoscale objects. Dentin hypersensitive of natural teeth have higher surface density of dentinal tubules and diameter and also larger than nonsensitive teeth. In dental nanorobots, we can use native biological materials, so it could selectively occlude specific tubules in a few minutes and offering patients a quick cure [1-3]. Even Orthodontic nanorobots could directly effect to the periodontal ligaments, and allowing rapid and painless tooth alignment in correct positioning within minutes to hours [4-7]. Nanorobotic manufacture of a biologically auto logous whole replacement tooth, that is, 'complete dentition replacement therapy' should become conveniently of a typical office visit with use of a desktop manufacturing facility, 
which would invent or fabricate the new tooth in the dentist's office not in the laboratory . In Nanorobotic analgesics, don't use needles, so there is a greater ability to control the analgesic effect, fast and reversible with avoid of side effects, to give patient comfort and also reduced nervousness [8-11]. Nanorobotic dentifrice (dentifrobots) take by mouthwash or toothpaste that could control all supragingival and sub gingival surfaces using once a day or more, metabolizing the organic matter into harmless and odorless vapors and prevent calculus debridement and plaque accumulation. With this kind of daily dental care available from an early age, we can prevent tooth decay and gingival diseases.

The international organization defines the robot as "an automatically controlled, reprogrammable, multipurpose manipulator with three or more axes." The institute of robotic in The United States Of America defines the robot as "a reprogrammable, multifunctional manipulator design to move material, parts, tools, or specialized devices through various programmed motions for the performance of variety of tasks". Robot manipulator is a collection of links that connect to each other by joints, these joints can be revolute and prismatic that revolute joint has rotary motion around an axis and prismatic joint has linear motion around an axis. Each joint provides one or more degrees of freedom (DOF). corresponds to the connection between two links pronding the required physical constraints on the relative motion between these two links. Most of joints is divided into three types; revolute joints that have one angular DOF, prisnratic joints which included one translate DOF and spherical joints that consisting of three DOF. Figures 1 to 3 show the revolute, prismatic and three degrees of freedom (spherical) joint.

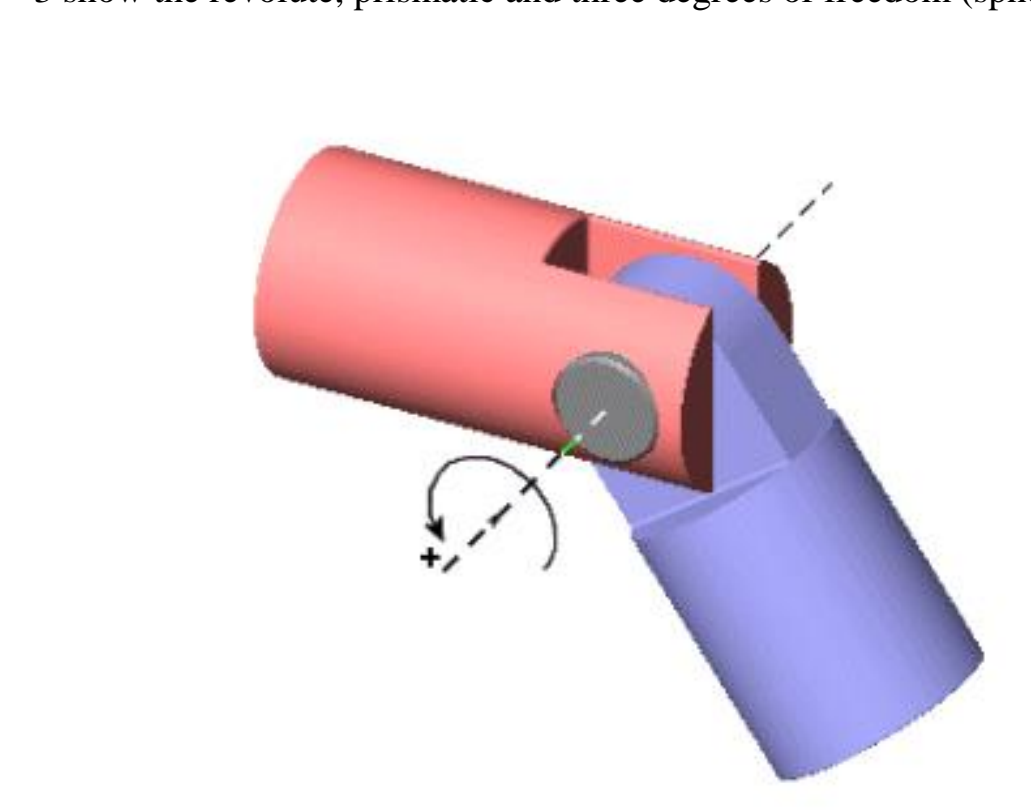

Figure 1. Revolute (1 DOF) Joint 


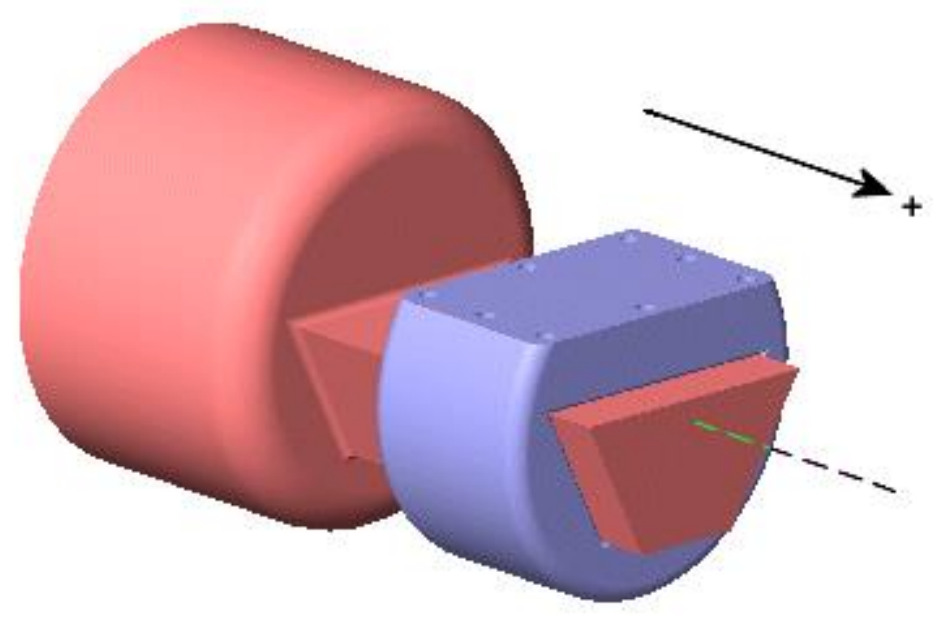

Figure 2. Prismatic (1 DOF) Jolint *

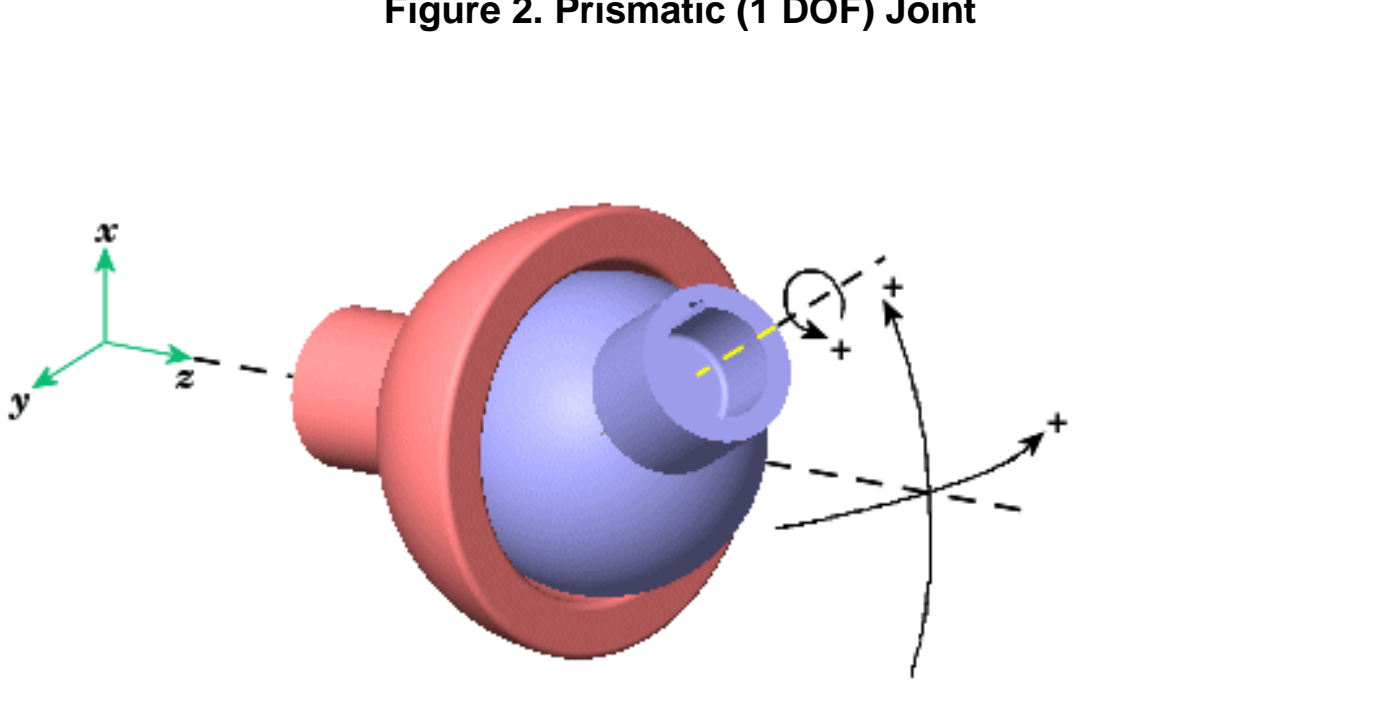

Figure 3. Three Degrees of Freedom (Spherical) Joint

Multi-degrees-of-freedom (DOF) actuators are wide used in a number of Industries. Currently, a significant number of the existing robotic actuators that can realize multiDOF motion are constructed using gear and linkages to connect several single-DOF motors in series and/or parallel. Not only do such actuators tend to be large in size and mass, buthey also have a decreased positioning accuracy due to mechanical deformation, friction and backlash of the gears and linkages. A number of these systems also exhibit singularities in their workspaces, which makes it virtually impossible to obtain uniform, high speed, and high-precision motion. For high precession trajectory planning and control, it is necessary to replace the actuator system made up of several single-DOF motors connected in series and/or parallel with a single multi-DOF actuator. The need for such systems has motivated years of research in the development of unusual, yet high performance actuators that have the potential to realize multi-DOF motion in a single joint. One such actuator is the spherical motor. Compared to conventional robotic manipulators that offer the same motion capabilities, the spherical motor possesses several advantages. Not only can the motor combine 3-DOF motion in a single joint, it has a large range of motion with no singularities in its workspace. The spherical motor is much simpler and more compact in design than most multiple single-axis robotic 
manipulators. The motor is also relatively easy to manufacture. The spherical motor have potential contributions to a wide range of applications such as coordinate measuring, object tracking, material handling, automated assembling, welding, and laser cutting. All these applications require high precision motion and fast dynamic response, which the spherical motor is capable of delivering. Previous research efforts on the spherical motor have demonstrated most of these features. These, however, come with a number of challenges. The spherical motor exhibits coupled, nonlinear and very complex dynamics. The design and implementation of feedback controllers for the motor are complicated by these dynamics. The controller design is further complicated by the orientation-varying torque generated by the spherical motor. Some of these challenges have been the focus of previous and ongoing research [12].

Controller is a device which can sense information from linear or nonlinear system to improve the systems performance. Several nonlinear systems are controlled by linear methodologies (e.g., Proportional-Derivative (PD) controller, Proportional- Integfal (PI) controller or Proportional- Integral-Derivative (PID) controller), but in presence of structural and unstructured uncertainties this technique has limitations. In medical industries and dental automation robot/joint are used in an unknown and unstructured environment, therefore strong mathematical tools used in new control methodologies to design nonlinear robust controller with an acceptable performance (e.g., mínimum error, good trajectory, disturbance rejection). Sliding modecontroller is an influential nonlinear controller to certain and uncertain systems which it is based on systen's dynamic model. This controller works very well in certain and partly uncertain condition. This controller has two important subparts, switching part and equivalent part. Switching part of controller is used to design suitable traeking performance based on very fast switching. This part has essential role to have a good trajectory performance in all joints. However this part is very important in uncertan condition but it is caused to chattering phenomenon in system performance. Chattering phenomenon can cause some important mechanical problems. The second subpart in sliding mode controller is equivalent part especially in uncertain condition. Sliding mod controller is a nonlinear model based controller and equivalent part is a dynamic formulation of system's dynamic that is used in control formulation of dental joint, which is used in control formulation to eliminate the decoupling and nonlinear term of dynamic parameters. Due to literature to reduce or eliminate the chattering two man methodologies are introduced:

Linear (saturation) boundary layer method

Nonlinear artificial intelligence based method

However, eliminating the switching discountinous function in sliding mode controller are used in many research but it can causes to lost the robustness of control and accuracy. Many researchers can reduce the chattering but they also lost the system stability based onmethods. In this research reduce or eliminate the chattering according to maintain the robustness is the main objective. Switching function is caused to chattering but it is one of the main parts to design robust and high speed sliding mode controller. In sliding mode controller, sliding surface slope $(\lambda)$ is the second factor to control the chattering, as a result the main task in the first objective is reduce or eliminate the chatering in sliding mode controller based on design parallel linear control methodology and discontinuous part. Sliding mode controller and linear control methodologies are robust based on Lyapunov theory, therefore; Lyapunov stability is proved in proposed chattering free sliding mode controller based on switching theory.

In this research, the robust challenge of dental joint is improve and discussed. This paper is organized as follows; section 2, is served as an introduction to the dynamic of three degrees of freedom spherical motor, intro to linear Controller. Part 3, introduces and describes the methodology algorithm. Section 4 presents the simulation results and discussion of this algorithm and the final section describe the conclusion. 


\section{Theory}

Dynamic and Kinematics Formulation of Spherical Motor: Dynamic modeling of spherical motors is used to describe the behavior of spherical motor such as linear or nonlinear dynamic behavior, design of model based controller such as pure sliding mode controller which design this controller is based on nonlinear dynamic equations, and for simulation. The dynamic modeling describes the relationship between motion, velocity, and accelerations to force/torque or current/voltage and also it can be used to describe the particular dynamic effects (e.g., inertia, coriolios, centrifugal, and the other parameters) to behavior of system. Spherical motor has nonlinear and uncertain dynamic parameters 3 degrees of freedom (DOF) motor.

The equation of a spherical motor governed by the following equation:

$$
H(q)\left[\begin{array}{c}
\ddot{\alpha} \\
\ddot{\beta} \\
\ddot{\gamma}
\end{array}\right]+B(q)\left[\begin{array}{c}
\dot{\alpha} \dot{\beta} \\
\dot{\alpha} \dot{\gamma} \\
\dot{\beta} \dot{\gamma}
\end{array}\right]+C(q)\left[\begin{array}{c}
\dot{\alpha}^{2} \\
\dot{\beta}^{2} \\
\dot{\gamma}^{2}
\end{array}\right]=\left[\begin{array}{c}
\tau_{x} \\
\tau_{y} \\
\tau_{z}
\end{array}\right]
$$

Where $\tau$ is actuation torque, $\mathrm{H}(\mathrm{q})$ is a symmetric and positive define juertia matrix, $\mathrm{B}(\mathrm{q})$ is the matrix of coriolios torques, $\mathrm{C}(\mathrm{q})$ is the matrix of centrifugal torques.

This is a decoupled system with simple second orden linear differential dynamics. In other words, the component $\ddot{q}$ influences, with a double integyator relationship, only the variable $q_{i}$, independently of the motion of the other parts. Therefore, the angular acceleration is found as to be:

$$
\ddot{\boldsymbol{q}}=\boldsymbol{H}^{-\mathbf{1}}(\boldsymbol{q}) \cdot\{\boldsymbol{\tau}-\{\boldsymbol{B}+\boldsymbol{C}\}\}
$$

This technique is very attractive from a control point of vew

Study of spherical motor is classified in o tyo main groups: kinematics and dynamics. Calculate the relationship between rigid bodies and final part without any forces is called Kinematics. Study of this part is pivotal to design with an acceptable performance controller, and in real situations and practical applications. As expected the study of kinematics is divided into two main parts. Corward and inverse kinematics. Forward kinematics has been used to find the position and orientation of task frame when angles of joints are known. Inverse kinematics has been used to find possible joints variable (angles) when all position and orientation of task frame be active.

The main target in forward kinematics is calculating the following function:

$$
\Psi(X, q)=0
$$

Where $\Psi()=.R^{n}$ is a nenlinear vector function, $X=\left[X_{1}, X_{2}, \ldots \ldots, X_{l}\right]^{T}$ is the vector of task space variables which generally task frame has three task space variables, three orientation, $q=\left[q_{1}, q_{2}, \ldots, q_{n}\right]^{T}$ is a vector of angles or displacement, and finally $n$ is the number of actuated joints. The Denavit-Hartenberg (D-H) convention is a method of drawing spherical motor free body diagrams. Denvit-Hartenberg (D-H) convention study is necessary to calculate forward kinematics in this motor.

A systematic Forward Kinematics solution is the main target of this part. The first step to compute Forward Kinematics (F.K) is finding the standard D-H parameters. The following steps show the systematic derivation of the standard D-H parameters.

Locate the spherical motor

Label joints

Determine joint rotation $(\theta)$

Setup base coordinate frames.

Setup joints coordinate frames.

Determine $\alpha_{i}$, that $\alpha_{i}$, link twist, is the angle between $Z_{i}$ and $Z_{i+1}$ about an $X_{i}$.

Determine $d_{i}$ and $a_{i}$, that $a_{i}$, link length, is the distance between $Z_{i}$ and $Z_{i+1}$ along $X_{i}$. $d_{i}$, offset, is the distance between $X_{i-1}$ and $X_{i}$ along $Z_{i}$ axis. 
Fill up the D-H parameters table. The second step to compute Forward kinematics is finding the rotation matrix $\left(R_{n}^{0}\right)$. The rotation matrix from $\left\{F_{i}\right\}$ to $\left\{F_{i-1}\right\}$ is given by the following equation;

$$
R_{i}^{i-1}=U_{i\left(\theta_{i}\right)} V_{i\left(\alpha_{i}\right)}
$$

Where $U_{i\left(\theta_{i}\right)}$ is given by the following equation;

$$
U_{i\left(\theta_{i}\right)}=\left[\begin{array}{ccc}
\cos \left(\theta_{i}\right) & -\sin \left(\theta_{i}\right) & 0 \\
\sin \left(\theta_{i}\right) & \cos \left(\theta_{i}\right) & 0 \\
0 & 0 & 1
\end{array}\right]
$$

and $V_{i\left(\alpha_{i}\right)}$ is given by the following equation;

$$
V_{i\left(\theta_{i}\right)}=\left[\begin{array}{ccc}
1 & 0 & 0 \\
0 & \cos \left(\alpha_{i}\right) & -\sin \left(\alpha_{i}\right) \\
0 & \sin \left(\alpha_{i}\right) & \cos \left(\alpha_{i}\right)
\end{array}\right]
$$

$$
\begin{aligned}
& \text { So }\left(R_{n}^{0}\right) \text { is given by } \\
& \boldsymbol{R}_{\boldsymbol{n}}^{\mathbf{0}}=\left(\boldsymbol{U}_{\mathbf{1}} \boldsymbol{V}_{\mathbf{1}}\right)\left(\boldsymbol{U}_{\mathbf{2}} \boldsymbol{V}_{\mathbf{2}}\right) \ldots \ldots \ldots\left(\boldsymbol{U}_{\boldsymbol{n}} \boldsymbol{V}_{\boldsymbol{n}}\right)
\end{aligned}
$$

The final step to compute the forward kinematics is calculate the transformation ${ }_{n}^{0} T$ by the following formulation [3]

$$
{ }_{n}^{0} T={ }_{1}^{0} T \cdot{ }_{2}^{1} T \cdot{ }_{3}^{2} T \ldots \ldots .{ }_{n}^{n-1} T=\left[\begin{array}{cc}
R_{n}^{0} & 0 \\
0 & 1
\end{array}\right]
$$

Linear Control Algorithm: Linear control theory is used in linear and nonlinear systems. This type of theory is used in industries, because design of this type of controller is simple than nonlinear controller. However this type of controller used in many applications but it cannot guarantee performance in complex systems. Simple linear controllers are including proportionat algorithm. Proportional-Derivative algorithm, Integral algorithm, Proportional-Integral algorithm and Proportional-Integral-Derivative algorithm.

Proportional Algorithm: It is used to responds immediately to difference of control input variables by immediately changing its influences variables, but this type of control is unable to eliminate the control input difference.

Proportional plus Derivative (PD) control: This type of linear controller is widely used in control process where the results are sensitive to exceeded of set point. This controller, like Proportional contyoller, has permanent variation in presence of selflimitation contro. In mathematically, the formulation of Proportional-Derivative part calculated as follows;

$$
U_{P D}=K_{p} \times e+K_{v}\left(\frac{d}{d t}\right)=K_{p} \times e+K_{v} \dot{e}
$$

The Derivative component in this type of methodology is used to cancel outs the change process variables change in presence of quick change in controllers input..

Integral (1) control: This category, integrate the input signal deviation over a period of time. This part of controller is used to system stability after a long period of time. In contrast of Proportional type of controller, this type of controller used to eliminate the deviation.

In mathematically, the formulation of integral part calculated as follows;

$$
I=\frac{1}{T} \int e \cdot d t=\sum e
$$

Proportional plus Integral (PI) control: According to integral type of controller, it takes relatively long time. The proportional type controller used to immediately response to the input variations. The proportional-integral (PI) controller has the advantages of both proportional and integral controller; it is rapid response to the input deviation as well as the exact control at the desired input.

$$
U_{P I}=K_{p} \times e+K_{i}\left(\frac{1}{T} \int e . d t\right)=K_{p} \times e+K_{i} \sum e
$$




\section{Methodology}

Sliding mode controller (SMC) is a powerful nonlinear controller which has been analyzed by many researchers especially in recent years. This theory was first proposed in the early 1950 by Emelyanov and several co-workers and has been extensively developed since then with the invention of high speed control devices The main reason to opt for this controller is its acceptable control performance in wide range and solves two most important challenging topics in control which names, stability and robustness. Sliding mode control theory for control of nonlinear system has been proposed in 1978 by Young to solve the set point problem $\left(\dot{\boldsymbol{q}}_{\boldsymbol{d}}=\mathbf{0}\right)$ by discontinuous method in the following form

$U_{(q, t)}= \begin{cases}U_{i}^{+}(q, t) & \text { if } S_{i}>0 \\ U_{i}^{-}(q, t) & \text { if } S_{i}<0\end{cases}$

where $\boldsymbol{S}_{\boldsymbol{i}}$ is sliding surface (switching surface), $\boldsymbol{i}=\mathbf{1}, \mathbf{2}, \ldots \ldots, \boldsymbol{n}$ for n-DOF dental joint, $\boldsymbol{U}_{\boldsymbol{i}}(\boldsymbol{q}, \boldsymbol{t})$ is the $\boldsymbol{i}^{\boldsymbol{t} \boldsymbol{h}}$ torque of joint. Sliding mode controller is divided into two main sub controllers: discontinues controller $\left(\boldsymbol{U}_{\text {dis }}\right)$ and equivalent controller $\left(\boldsymbol{U}_{\boldsymbol{e q}}\right)$.

Discontinues controller causes an acceptable tracking performance at the expense of very fast switching. Conversely in this theory good trajectory following is based on fast switching, fast switching is caused to have system instability and chattering phenomenon. Fine tuning the sliding surface slope is based on nonlinear equvalent part However, this controller is used in many applications but, pure ficting mode controller has chattering phenomenon challenge. Chattering phenomenon can causes some problems such as saturation and heats the mechanical parts of drivers.

In boundary layer saturation method, the basic idea is the discontinuous method replacement by saturation (linear) method with small neighborhood of the switching surface. This replacement caused to increase the enor performance against with the considerable chattering reduction. Désign a robust controller for dental joint is essential because this type of joint has highly nonlinear dynamic parameters. Consider a nonlinear single input dynamic systemis defined by:

$x^{(n)}=f(\vec{x})+b(\vec{x}) u$

$\tilde{x}=x-x_{d}=\left[\tilde{x}, \ldots, \tilde{x}^{(n-1)}\right]^{T}$

A time-varying sliding surfaces $s(x, t)$ in the state space $\boldsymbol{R}^{\boldsymbol{n}}$ is given by:

$$
s(x, t)=\left(\frac{d}{d t}+\lambda\right)^{n-1} \vec{x}=0
$$

where $\lambda$ is the posit ye constant. To further penalize tracking error, integral part can be used in sliding surface part as follows:

$$
s(x, t)=\left(\frac{d}{d t}+\lambda\right)^{n-1}\left(\int_{0}^{t} \tilde{x} d t\right)=0
$$

The nam target in this methodology is kept the sliding surface slope $\boldsymbol{s}(\boldsymbol{x}, \boldsymbol{t})$ near to the zero Therefore, one of the common strategies is to find input $\boldsymbol{U}$ outside of $\boldsymbol{s}(\boldsymbol{x}, \boldsymbol{t})$.

$\frac{1}{2 d t} s^{2}(x, t) \leq-\zeta|s(x, t)|$

where $\zeta$ is positive constant.

If $\mathrm{S}(0)>0 \rightarrow \frac{d}{d t} S(t) \leq-\zeta$

To eliminate the derivative term, it is used an integral term from $\mathrm{t}=0$ to $\mathrm{t}=\boldsymbol{t}_{\text {reach }}$

$\int_{t=0}^{t=t_{\text {reach }}} \frac{d}{d t} S(t) \leq-\int_{t=0}^{t=t_{\text {reach }}} \eta \rightarrow S\left(t_{\text {reach }}\right)-S(0) \leq-\zeta\left(t_{\text {reach }}-0\right)$

Where $t_{\text {reach }}$ is the time that trajectories reach to the sliding surface so, suppose $\mathrm{S}\left(t_{\text {reach }}=0\right)$ defined as 


$$
0-S(0) \leq-\eta\left(t_{\text {reach }}\right) \rightarrow t_{\text {reach }} \leq \frac{S(0)}{\zeta}
$$

and

$$
\begin{aligned}
& \text { if } S(0)<0 \rightarrow 0-S(0) \leq-\eta\left(t_{\text {reach }}\right) \rightarrow S(0) \leq-\zeta\left(t_{\text {reach }}\right) \rightarrow t_{\text {reach }} \\
& \leq \frac{|\boldsymbol{S}(\mathbf{0})|}{\eta} \\
& \text { if } S_{t_{\text {reach }}}=S(0) \rightarrow \operatorname{error}\left(x-x_{d}\right)=0
\end{aligned}
$$

suppose $S$ is defined as

$$
s(x, t)=\left(\frac{d}{d t}+\lambda\right) \quad \widetilde{x}=\left(\dot{x}-\dot{x}_{d}\right)+\lambda\left(x-x_{d}\right)
$$

The derivation of S, namely, $\dot{S}$ can be calculated as the following;

$\dot{S}=\left(\ddot{x}-\ddot{x}_{d}\right)+\lambda\left(\dot{x}-\dot{x}_{d}\right)$

suppose the second order system is defined as;

$\ddot{x}=f+u \rightarrow \dot{S}=f+U-\ddot{x}_{d}+\lambda\left(\dot{x}-\dot{x}_{d}\right)$

Where $\boldsymbol{f}$ is the dynamic uncertain, and also since $S=$ and $\dot{S}=0$, to have the best approximation, $\widehat{\boldsymbol{U}}$ is defined as $\widehat{U}=-\widehat{f}+\ddot{x}_{d}-\lambda\left(\dot{x}-\dot{x}_{d}\right)$

A simple solution to get the sliding condition when the dynamic parameters have uncertainty is the switching control law:

$\boldsymbol{U}_{\text {dis }}=\widehat{U}-K(\vec{x}, t) \cdot \operatorname{sgn}(\boldsymbol{s})$

where the switching function $\boldsymbol{s} g \boldsymbol{n}(\boldsymbol{S})$ is defined as

$\operatorname{sgn}(s)= \begin{cases}1 & s>0 \\ -1 & s<0 \\ 0 & s=0\end{cases}$

and the $\boldsymbol{K}(\overrightarrow{\boldsymbol{x}}, \boldsymbol{t})$ is the positive constant.

$\frac{1}{2} \frac{d}{d t} s^{2}(x, t)=\dot{S} S=[f-\hat{f}-K \operatorname{sgn}(s)] \cdot S=(f-\widehat{f}) \cdot S-K|S|$

The sliding surface can becalculated as

$s(x, t)=\left(\frac{d}{d t}+\lambda\right)^{2}\left(\int_{0} \tilde{x} d t\right)=\left(\dot{x}-\dot{x}_{d}\right)+2 \lambda\left(\dot{x}-\dot{x}_{d}\right)-\lambda^{2}\left(x-x_{d}\right)$

in this method the approximation of $\boldsymbol{U}$ is computed as [6]

$\widehat{U}=-\widehat{f}+\ddot{x}_{d}-2 \lambda\left(\dot{x}-\dot{x}_{d}\right)+\lambda^{2}\left(x-x_{d}\right)$

$$
U=U_{e q} U_{d i s}
$$

$U_{Q q}=\left[H^{-1}(B+C)+\dot{S}\right] H$

$U_{d i s}=K \cdot \operatorname{sgn}(S)$

The combination of proportional (P) component, integral (I) component with a derivative (D) controller offered advantages in each case. This type of controller has rapid response to the input deviation, the exact control at the desired input as well as fast response to the disturbances. The PID controller takes the error between the desired joint variables and the actual joint variables to control. A proportional-derivative integral control system can easily be implemented. This method does not provide sufficient 
control for systems with time-varying parameters or highly nonlinear systems. The formulation of PID controller calculated as follows;

$$
U_{P I D}=K_{p} \times e+K_{i}\left(\frac{1}{T} \int e . d t\right)+K_{v}\left(\frac{d e}{d t}\right)=K_{p} \times e+K_{i} \sum e+K_{v} \dot{e}
$$

Proportional-Integral-Derivative (PID) controller has rapid response to the input deviation, the exact control at the desired input as well as fast response to the disturbances. The PID controller takes the error between the desired joint variables and the actual joint variables to control the three dimension of joint. The equation of PID controller for control of 3 degrees of freedom joint is;

$$
\left[\begin{array}{l}
\widehat{\tau_{1}} \\
\widehat{\tau_{2}} \\
\widehat{\tau_{3}}
\end{array}\right]=\left[\begin{array}{l}
K_{i 1} \sum e_{1}+K_{v 1} \dot{e}_{1}+K_{p 1} e_{1} \\
K_{i 2} \sum e_{2}+K_{v 2} \dot{e}_{2}+K_{p 2} e_{2} \\
K_{i 3} \sum e_{3}+K_{v 3} \dot{e}_{3}+K_{p 3} e_{3}
\end{array}\right]
$$

Where $e=q_{d}-q_{a}, q_{d}$ is desired joint variable and $q_{a}$ is actual joint variable.

In PID controller the control law is given by the following equation;

$\tau=K_{p} e+K_{v} \dot{e}+K_{i} \sum e$

Where $\boldsymbol{e}=\boldsymbol{q}_{\boldsymbol{i}_{\boldsymbol{d}}}-\boldsymbol{q}_{\boldsymbol{i}_{\boldsymbol{a}}}$

In this theory $\boldsymbol{K}_{\boldsymbol{p}}, \boldsymbol{K}_{\boldsymbol{i}}$ and $\boldsymbol{K}_{v}$ are positive constant. To show this controller is stable and achieves zero steady state error, the Lyapunov unction is,introduced;

$V=\frac{1}{2}\left[\dot{q}^{T} A(q) \dot{q}+e^{T} K_{p} e\right]=$

$\frac{1}{2} \frac{d}{d t}\left[\dot{q}^{T} A \dot{q}\right]=\dot{q} \tau$

If the conversation energy is written by the following form:

$$
\frac{1}{2} \frac{d}{d t}\left[\dot{q}^{T} A \dot{q}\right]=\dot{q} \tau
$$

Where $(\dot{q} \tau)$ shows the power inputs from actuator and $\frac{1}{2} \frac{d}{d t}\left[\dot{q}^{T} A d\right]$ is the derivatie of the robot kinematic energy.

$\dot{V}=\dot{q}^{T}\left[\tau+K_{p} e\right]$

Based on $\boldsymbol{\tau}=-\boldsymbol{K}_{\boldsymbol{p}_{\boldsymbol{i}}} \boldsymbol{e}-\boldsymbol{K}_{\boldsymbol{v}_{\boldsymbol{i}}} \dot{\boldsymbol{e}}-\boldsymbol{K}_{\boldsymbol{i}} \sum \boldsymbol{e}$, we can write:

$\dot{V}=\dot{q}^{T} K_{p} \dot{q} \leq \mathbf{0}$

$$
\dot{q}=\mathbf{0} \rightarrow \ddot{q}=\mathbf{0} \rightarrow \ddot{q}=A^{-1} K_{p} e \rightarrow e=0
$$

In this state, the actual trajectories converge to the desired state.

To eliminate the chattering boundary layer method is introduced. In this method the researcher introduced saturation function in the sliding mode control law instead of the switching (sign) function. The saturation (linear) method with small neighborhood of the switching surface is calculated as: 


$$
\boldsymbol{B}(\boldsymbol{t})=\{\boldsymbol{x},|\boldsymbol{S}(\boldsymbol{t})| \leq \emptyset\} ; \varnothing>0
$$

where $\emptyset$ is the boundary layer thickness. Consequently the sliding mode function:

$$
U=K(\vec{x}, t) \cdot \operatorname{Sat}(S / \varnothing)
$$

While saturation function formulation $(\boldsymbol{S a t}(\boldsymbol{S} / \varnothing))$ is as follows

$$
\operatorname{sat}(s / \varnothing)=\left\{\begin{array}{cc}
1 & (s / \varnothing>1) \\
-1 & (s / \varnothing<-1) \\
\boldsymbol{s} / \varnothing & (-1<s / \varnothing<1)
\end{array}\right.
$$

Considering the above points, to reduce chattering phenomenon in sliding mode controller based on saturation function, the following formulation is used

$U=U_{e q}+U_{s a t}$

and $\boldsymbol{U}_{\boldsymbol{s a t}}$ is computed as

$U_{\text {sat }}=K \cdot \operatorname{sat}(S / \varnothing)$

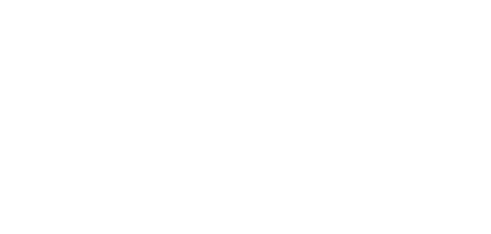

This method reduces or eliminates the chattering but $\mathbb{C}$ has challenge to proof the robust and stability.

To reduce the chattering in presence of switching unctions; linear controller is added to discontinuous part of sliding mode Controller Lmear controller is type of stable controller as well as conventionalssiding mode controller. In proposed methodology PD, PI or PID linear controller is used in parallel with discontinuous part to reduce the role of sliding surface slope as a main coefficient The lormulation of new chattering free sliding mode controller for dentahojint is;

$\boldsymbol{U}=U_{\text {eq }}+U_{\text {dis }- \text { new }}$

The new switching discontinuous part is introduced by $\boldsymbol{U}_{\text {dis-new }}$ a6nd this item is the important facto to resistance and robust in this controller. In PD sliding surface, the change of sliding surface calculated as;

$$
S_{P D}=\lambda e+\dot{e} \rightarrow \dot{S}_{P D}=\lambda \dot{e}+\ddot{e}
$$

The discontinuous switching term $\left(\boldsymbol{U}_{\text {dis }}\right)$ is computed as

$$
U_{\text {dis-new }}=K_{a} \cdot \operatorname{sgn}(S)+K_{b} \cdot S_{P I D}
$$

$$
U_{d i s-P D-n e w}=K_{a} \cdot \operatorname{sgn}(\lambda e+\dot{e})+K_{b} \cdot\left(\lambda e+\dot{e}+\left(\frac{\lambda}{2}\right)^{2} \sum e\right)
$$

Based on proof of robust and stability, this type of method is more robust and stable than boundary layer sliding mode controller.

\section{Results and Discussion}

In result and discussion section, conventional SMC, boundary layer SMC and proposed methodology have been compared for dental joint.

Comparison of the Tracking Data and Information: the trajectory following for saturation boundary layer sliding mode controller and proposed method are compared in 
this section. Sliding mode controller has high frequency oscillation chattering. To chattering attenuation linear boundary layer saturation function is replaced by switching sign function in sliding mode controller. However boundary layer method can reduce the chattering but this method has two challenges: proof the stability and quality of performance. To solve these two challenges, parallel linear sliding mode controller is used. Based on the following Figure, eliminating the sign function in sliding mode controller using saturation function caused to eliminate the chattering however, robustness of control and accuracy are lost. The trajectory following and chattering elimination is illustrated in Figure 4. According to Figure 4, parallel linear sliding mode controller (proposed-SMC) is faster than saturation sliding mode controller because the rise time in parallel sliding mode controller is 0.55 second and in saturation sliding mode controller is 3.14 second. In error point of view, parallel linear SMC is better than boundary layer SMC. However, boundary layer sliding mode controller can reduce the chattering but this method is caused to increase undershoot and error. According to Figure 4, paralleNlinear sliding mode controller has accurate trajectory response and it can eliminate the chattening as well as reduce the error.

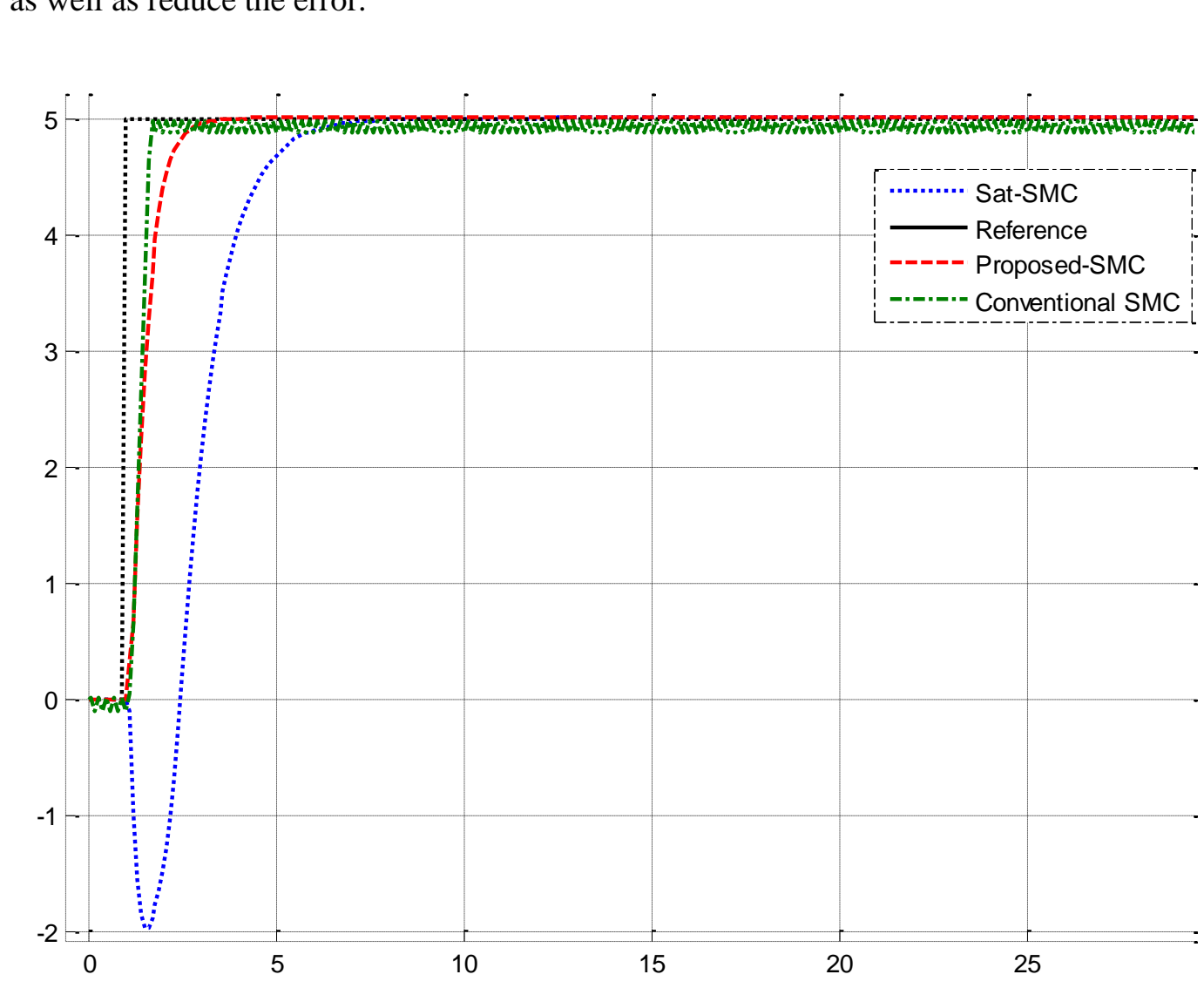

\section{Figure 4. Conventional SMC, Saturation SMC and Parallel Linear SMC (Proposed-SMC)}

Comparison the sliding surface $(\boldsymbol{S})$ : sliding surface is a linear function that is defined based on PD, PI or PID formulation. Sliding surface in sliding mode controller is compared to examine the stability and the size of chattering. Figure 5 shows the sliding surface in conventional SMC, boundary layer sliding mode controller and parallel linear sliding mode controller (proposed-SMC). According to the following graph, the speed of parallel linear SMC is faster than saturation boundary layer SMC. 


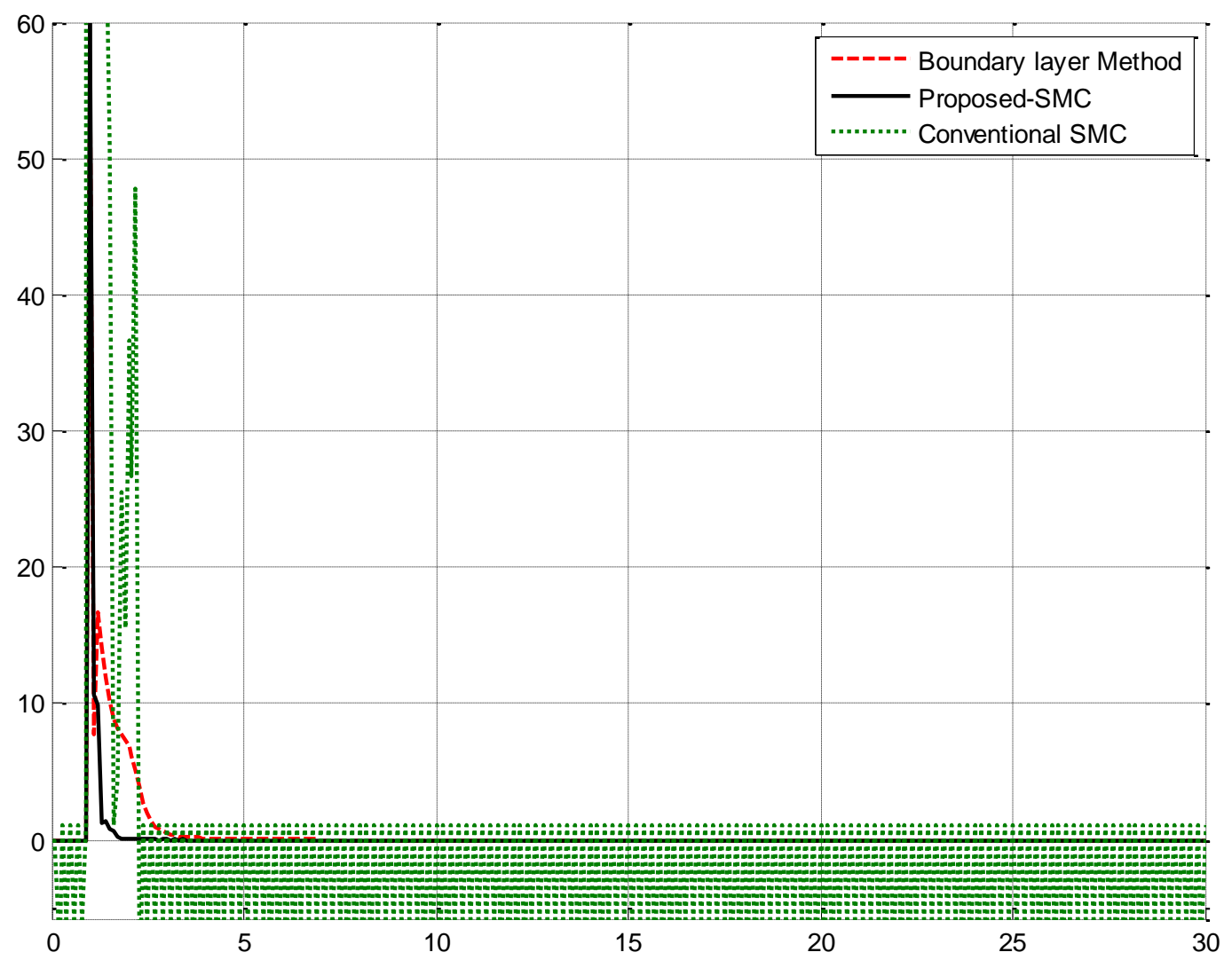

Figure 5. Comparison of Sliding Surface: Conventional SMC, Boundary Layer SMC and Parallel Linear SMC (Proposed SMC)

Based on Figure 5 sliding surface of boundary layer sliding mode controller and parallel linear sliding mode controllers are spike free but the speed of boundary layer SMC is slower then proposed method and conventional SMC.

Comparison the disturbance rejection: the power of disturbance rejection is very important to robust checking in any controllers. In this section trajectory accuracy are test under uncertainty condition. To test the disturbance rejection band limited white noise with $30 \%$ amplitude is applied to conventional SMC, parallel linear sliding mode controller (proposed SMC) and boundary layer sliding mode controller. In Figure 6, trajectory accuracy is shown. 


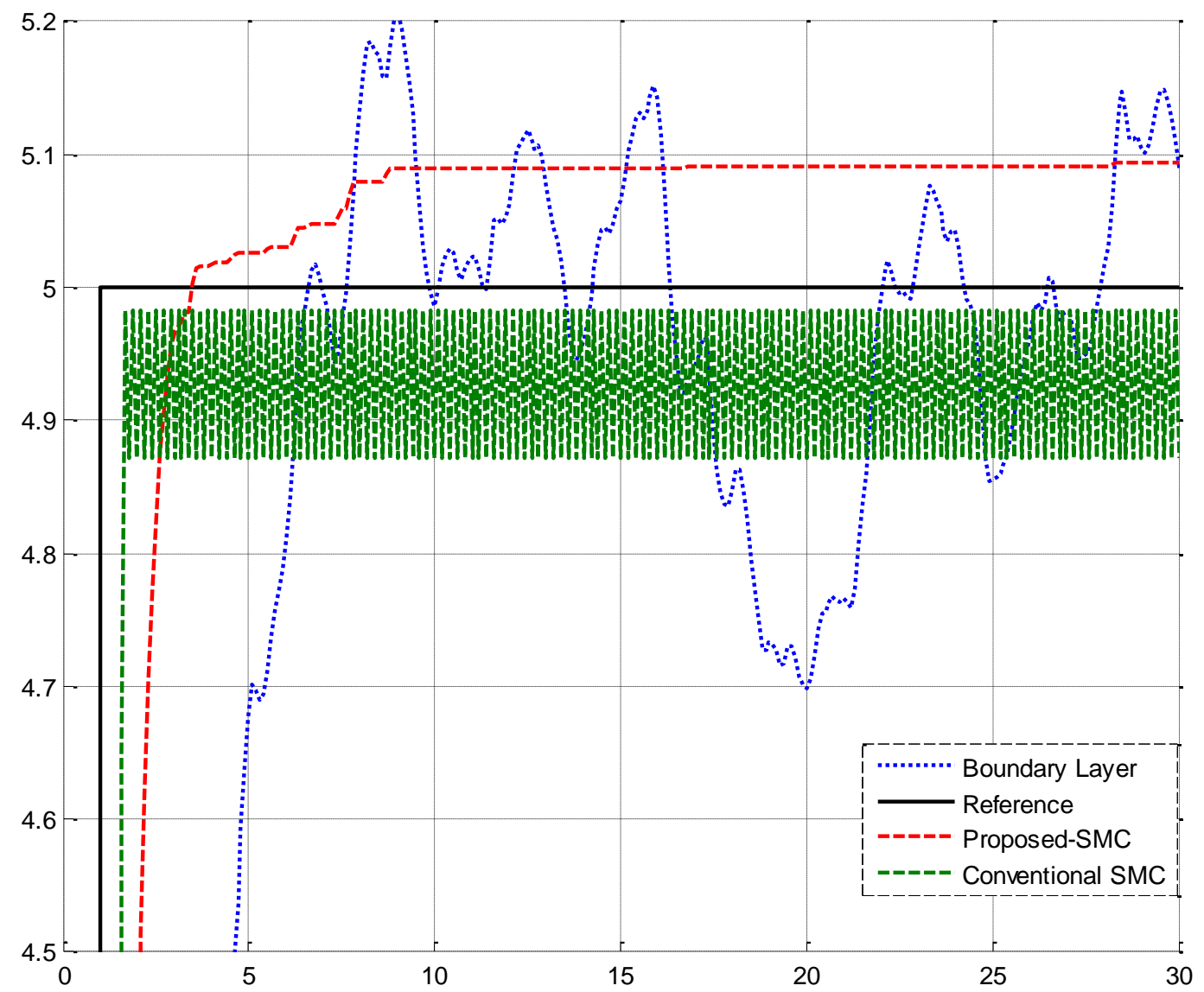

Figure 6. Comparison of Disturbance Rejection: Conventional SMC, Parallel Linear SMC (Proposed-SMC) and Boundary Layer Method

According to above grapb, however parallel linear sliding mode controller has suitable steady state error in presence of uncertainty but it is more robust than boundary layer sliding mode controller. Boundary layers sliding mode controller and conventional SMC have fluctuations in presence of external disturbance.

\section{Conclusion}

In this research, chatering free model base sliding mode controller is recommended for three degrees of freedom dental joint. Conventional sliding mode controller (SMC) is a nonlinear stable, robust and reliable controller. Chattering phenomenon is the main challenge m this type of control algorithm. To solve this challenge and improve the stability and robustness linear type algorithm is recommended. The simulation results show that the proposed controller works well in various situations. This type of method is the best robust method to eliminate the chattering in presence of uncertainty with swiching function. Based on result and discussion, proposed method can eliminate chattering in certain and uncertain condition. This controller has been reduced the noise oscillation in presence of uncertainties and $30 \%$ external disturbance.

\section{Acknowledgment}

This work was supported by the Iranian Institute of Advance Science and Technology Program of Iran under grant no. 2013-Persian Gulf-2A.

Project Title: Design High Presidion and Fast Dynamic Controller for Multi Degrees of Freedom 
Iranian center of Advance Science and Technology (IRAN SSP) is one of the independent research centers specializing in research and training across of Control and Automation, Electrical and Electronic Engineering, and Mechatronics \& Robotics in Iran. At IRAN SSP research center, we are united and energized by one mission to discover and develop innovative engineering methodology that solve the most important challenges in field of advance science and technology. The IRAN SSP Center is instead to fill a long-standing void in applied engineering by linking the training a development function one side and policy research on the other. This center divided into two main units:

Education unit

Research and Development unit

Please follow IRANSSP research and training group: http://iranssp.org/english/

\section{References}

[1] G. I.Vachtsevanos, K.Davey and K. M. Lee, "Development of a Novel Intelligent Robotic Manipurator", IEEE Control System Magazine, (1987), pp. 9-15.

[2] K. Davey, G. I.Vachtsevanos and R. Powers, "An analysis of Fields and Torques in Spherical Induction Motors", IEE Transactions on Magnetics, vol. MAG-23, (1987), pp. 273-282.

[3] A. Foggia, E. Oliver and F.Chappuis, "New Three Degrees of Freedom Electromagnetic Actuator", Conference Record -lAS Annual Meeting, New York, vol 35, (1988).

[4] K. M. Lee, G.Vachtsevanos and C-K. Kwan, "Development of a Spherical Wrist Stepper Motor", Proceedings of the 1988 IEEE lntemational Conference on Robotics and Autonation, Philadelphia, PA. April 26-29.

[5] K. M. Lee and I. Pei, "Kinematic Analysis of a Three.Degree^of-Ereedom Spherical Wrist Actuator", The Fifth International Conference on Advanced Robotics, Italy, (1991).

[6] I. Wang, G. Jewel and D. Howe, "Modeling of a Novel Spherical Pennanent Magnet Actuator", Proceedings of IEEE International Conference on Robotics and Automation, Albuquerque, New Mexico, (1997), pp 1190-1195.

[7] I. Wang, G. Jewel and D. Howe, "Anarysis, Design and Control of a Novel Spherical Pennanent Magnet Actuator", lEE Proceedings on Electrical Power Applications, vol. 154, no. 1, (1998).

[8] G. S. Chirikjian and D.Stein, "Kinematic Design and Commutation of a Spherical Stepper Motor", IEEEIASME Transactions on Mechatronics. Piscataway, New Jersey, vol. 4, no. 4, (1999), pp. 342-353.

[9] K. Kahlen and R. W. De Don cker, "CW'Kent Regulators for Multi-phase Pennanent Magnet Spherical Machines”, Industry Applications Conference Record of the 2000 IEEE, vol. 3, (2000), pp. 2011-2016.

[10] K. M. Lee, I. Pei and U. Gilboa, "Onthe Development of a Spherical Wrist Actuator", Proceedings of the 16th NSF Conference on Manufacturing Systems Research, Tempe AZ, (1990).

[11] C. Yang and Y S. Back, "Design and Control of the 3-dcgn:es of freedom actuator by Controlling the Electromagnetic Force" IEEE Transactions on Magnetics, (1999), pp. 3607-3609.

[12] A. Siahbazi A. Barzegar, M. Vosoogh, A. Majid Mirshekaran and S. Soltani, "Design Modified Sliding Mode Controller with Parahlel Fuzzy Inference System Compensator to Control of Spherical Motor", IJISA, DOI: $10.5815 / \mathrm{g}$ isa.2014.03.02, vol. 6, no. 3, pp.12-25, (2014).

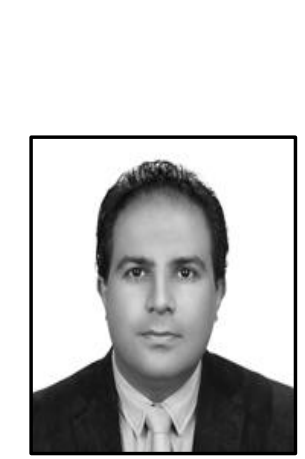

\section{Authors}

Farzin Piltan, he is an outstanding scientist in the field of Electronics and Control engineering with expertise in the areas of nonlinear systems, robotics, and microelectronic control. Mr. Piltan is an advanced degree holder in his field. Currently, Mr. Piltan is the Head of Mechatronics, Intelligent System, and Robotics Laboratory at the Iranian Institute of Advanced Science and Technology (IRAN SSP). Mr. Piltan led several high impact projects involving more than 150 researchers from countries around the world including Iran, Finland, Italy, Germany, South Korea, Australia, and the United States. Mr. Piltan has authored or co-authored more than 140 papers in academic journals, conference papers and book chapters. His papers have been cited at least 3900 times by independent and dependent researchers from around the world including Iran, Algeria, 
Pakistan, India, China, Malaysia, Egypt, Columbia, Canada, United Kingdom, Turkey, Taiwan, Japan, South Korea, Italy, France, Thailand, Brazil and more. Moreover, Mr. Piltan has peer-reviewed at least 23 manuscripts for respected international journals in his field. Mr. Piltan will also serve as a technical committee member of the upcoming EECSI 2015 Conference in Indonesia. Mr. Piltan has served as an editorial board member or journal reviewer of several international journals in his field as follows: International Journal of Control And Automation (IJCA), Australia, ISSN: 2005-4297, International Journal of Intelligent System and Applications (IJISA), Hong Kong, ISSN:2074-9058, IAES International Journal Of Robotics And Automation, Malaysia, ISSN:2089-4856, International Journal of Reconfigurable and Embedded Systems, Malaysia, ISSN:2089-4864.

Mr. Piltan has acquired a formidable repertoire of knowfedge and skills and established himself as one of the leading youngscientists in his field. Specifically, he has accrued expertise in the design and implementation of intelligent controk in nonlineary systems. Mr. Piltan has employed his remarkable-expertise in these areas to make outstanding contributions as detailed follows:Nonlinear control for industrial robot manipulator (2010-IRAN SSP), Intelligent Tuning The Rate Of Fuel Ratio In Internal Combustion Engine (2011IRANSSP), Design High Precision and Fast Dynamic Controller For Multi-Degrees Of Freedom Actuator (2013-IRANSSP), Research on Full Digital Controlfor Nonlinea Systems (2011-IRANSSP), MicroElectronic Based Intelligent Nonlinear Controller (2015-IRANSSP), Active Robot Controller for Dental Automation (2015-IRANSSP), Design a Micro-Electronio Based Nonlinear Controller for First Order Delay System (2015-1RANSSP).

The above original accomplishments clearly demonstrate that Mr. Piltan has performed original research and that he has gained a distinguished reputation as an outstanding scientist in the field of electronics and control engineering. Mr. Piltan has a tremendous and unique est of skills, knowledge and background for his current and future kor. He possesses a rare combination of academic knowledge and practical skills that are highly valuable for his work. In 2011, he pablished 28 first author papers, which constitute about 30\% of papers published by the Department of Electrical and Electronic Engineering at University Putra Malaysia. Additionally, his 28 papers represent about $6.25 \%$ and $4.13 \%$ of all control and system papers published in Malaysia and Iran, respectively, in 2011.

Meysam Esmaeili, he is currently research assistant at Institute of Advance Science and Technology, Research and Development Center, IRAN SSP. He has been working at "Design High Precision and Fast Dynamic Controller for Multi-Degrees of Freedom Actuator for Experimental Research and Education" project at Iranian Institute of Advance Science and Technology, Sanaat Kade Sabz Passargad Research Center (IRAN SSP) as "Pre-PhD Student Researcher"of a research team composed of 21 researchers since Feb. 2013 to Feb. 2014. He has had the main roles in initiation and development of this project which has resulted thus far in three scientific publications up to now. He has been working at "Research of Full Digital Control for 


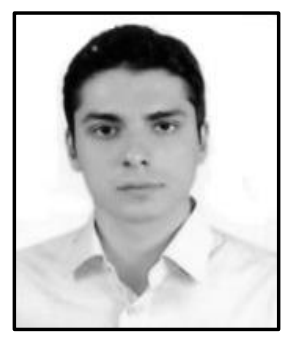

Nonlinear System for Experimental Research and Education" project at Iranian Institute of Advance Science and Technology, Sanaat Kade Sabz Passargad Research Center (IRAN SSP) as "research assistant scholar" of a research team composed of 27 researchers since Feb. 2014 to date. His current research interests are nonlinear control, artificial control system and design FPGA-based controller.

Mohammad Ali Tayebi, he is currently research assistant at Institute of Advance Science and Technology, Research and Development Center, IRAN SSP. He has been working at "Design High Precision and Fast Dynamic Controller for Multi-Degrees of Freedom Actuator for Experimental Research and Education" project at Iranian Institute of Advance Science and Technology, Sanaat Kade Sabz Passargad Research Center (IRAN SSP) as "Pre-PhD Student Researcher"of a research team composed of 21 researchers stince Feb. 2013 to Feb. 2014. He has had the main roles in jinitiat on and development of this project which has resulted thu far in three scientific publications up to now. He has beên working at "Research of Full Digital Control for Nonlinear System for Experimental Research and Education" project at Iranian Insticute of Advance Science and Technology, Sanaat Kade Sabz Passargad Research Center (IRAN SSP) as "research assistant scholar" of a research team composed of 27 researchers since Fe6 2014 to date. His current research interests are ronlinear control, artificial, control system and design FPGA-based controller.

Mahsa Pilan, she is currently research assistant at Institute of

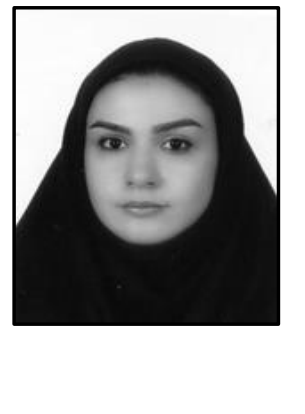
Advance Science and Technology, Research and Development Center, IRAN SSP She has been working at "DesignHigh Precision and Fast Dynamic Controller for Multi-Degrees of Freedom Actuator for Experimental Research and Education" project at Iranian Institute of Advance Seience and Technology, Sanaat Kade Sabz Passargad Research Center (IRAN SSP) as "Pre-PhD Student Researcher" of a research team composed of 21 researchers since Feb. 2013 to Feb. 2014. She has had the main roles in initiation and development of this project which has resulted thus far in three scientific publications up to now. She has been working at "Research of Full Digital Control for Nonlinear System for Experimental Research and Education" project at Iranian Institute of Advance Science and Technology, Sanaat Kade Sabz Passargad Research Center (IRAN SSP) as "research assistant scholar" of a research team composed of 27 researchers since Feb. 2014 to date. Her current research interests are nonlinear control, artificial control system and design FPGA-based controller.

Mojtaba Yaghoot, he is currently research assistant at Institute of Advance Science and Technology, Research and Development Center, IRAN SSP. He has been working at "Design High Precision and Fast Dynamic Controller for Multi-Degrees of Freedom Actuator for Experimental Research and Education" project at Iranian Institute of Advance Science and Technology, Sanaat Kade Sabz Passargad Research Center (IRAN SSP) as "Pre-PhD Student Researcher"of a research team composed of 21 researchers since Feb. 2013 to Feb. 


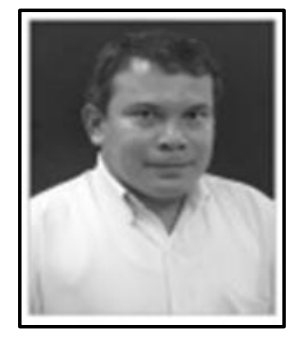

2014. He has had the main roles in initiation and development of this project which has resulted thus far in three scientific publications up to now. He has been working at "Research of Full Digital Control for Nonlinear System for Experimental Research and Education" project at Iranian Institute of Advance Science and Technology, Sanaat Kade Sabz Passargad Research Center (IRAN SSP) as "research assistant scholar" of a research team composed of 27 researchers since Feb. 2014 to date. His current research interests are nonlinear control, artificial, control system and design FPGA-based controller.

Nasri Sulaiman, he is a Senior Lecturer in the Department Electrical and Electronic Engineering at the Universiti Purta Malaysia (UPM), which is one of the leading research universities in Malaysia. $\mathrm{He}$ is a supervisor and senior researcher at research and training center called, Iranian Institute of Advanced Science and technology (Iranssp) since 2012. He obtained his M.Sc. from the University of Southampton (UK), and Ph.D. in Microelectronics from the University of Edinburgh (UK). He has published more than 80 technical papers related to control and system enginee ing, including several co-authored papers with Min. Piltan. He has been invited to present his research at numerous national and international conferences. He has supervised many graduate students at doctoral and masters level. He is an outstanding scientist in the field of MicroElectronics.

Dr. Nasri Sulainan advisor and supervisor of several high impact projects involving more than 150 researchers from countries around the world including Iran, Malaysia, Finland, Italy, Germany, South Korea, Australia, and the United States. Dr. Nasri Sulaiman has authored or co-authored more than 80 papers in academic journals, conference papers and book chapters. His papers have been cited at least 3000 times by independent and dependent researchers from around the world including Iran, Algeria, Pakistan, India, China, Málaysia, Egypt, Columbia, Canada, United Kingdom, Turkey, Taiwan, Japan, South Korea, Italy, France, Thailand, Brazil and more

Dr. Nasri Sulaiman has employed his remarkable expertise in these areas to make outstanding contributions as detailed below:

Design of a reconfigurable Fast Fourier Transform (FFT)

Processor using multi-objective Genetic Algorithms (2008-UPM)

Power consumption investigation in reconfigurable Fast Fourier Transform (FFT) processor (2010-UPM)

Crest factor reduction And digital predistortion Implementation in Orthogonal frequency Division multiplexing (ofdm) systems (2011UPM)

High Performance Hardware Implementation of a Multi-Objective Genetic Algorithm, (RUGS), Grant amount RM42,000.00, September (2012-UPM)

Nonlinear control for industrial robot manipulator (2010-IRAN SSP)

Intelligent Tuning The Rate Of Fuel Ratio In Internal Combustion Engine (2011-IRANSSP)

Design High Precision and Fast Dynamic Controller For MultiDegrees Of Freedom Actuator (2013-IRANSSP) 
Research on Full Digital Control for Nonlinear Systems (2011IRANSSP)

Micro-Electronic Based Intelligent Nonlinear Controller (2015IRANSSP)

Active Robot Controller for Dental Automation (2015-IRANSSP) Design a Micro-Electronic Based Nonlinear Controller for First Order Delay System (2015-IRANSSP)

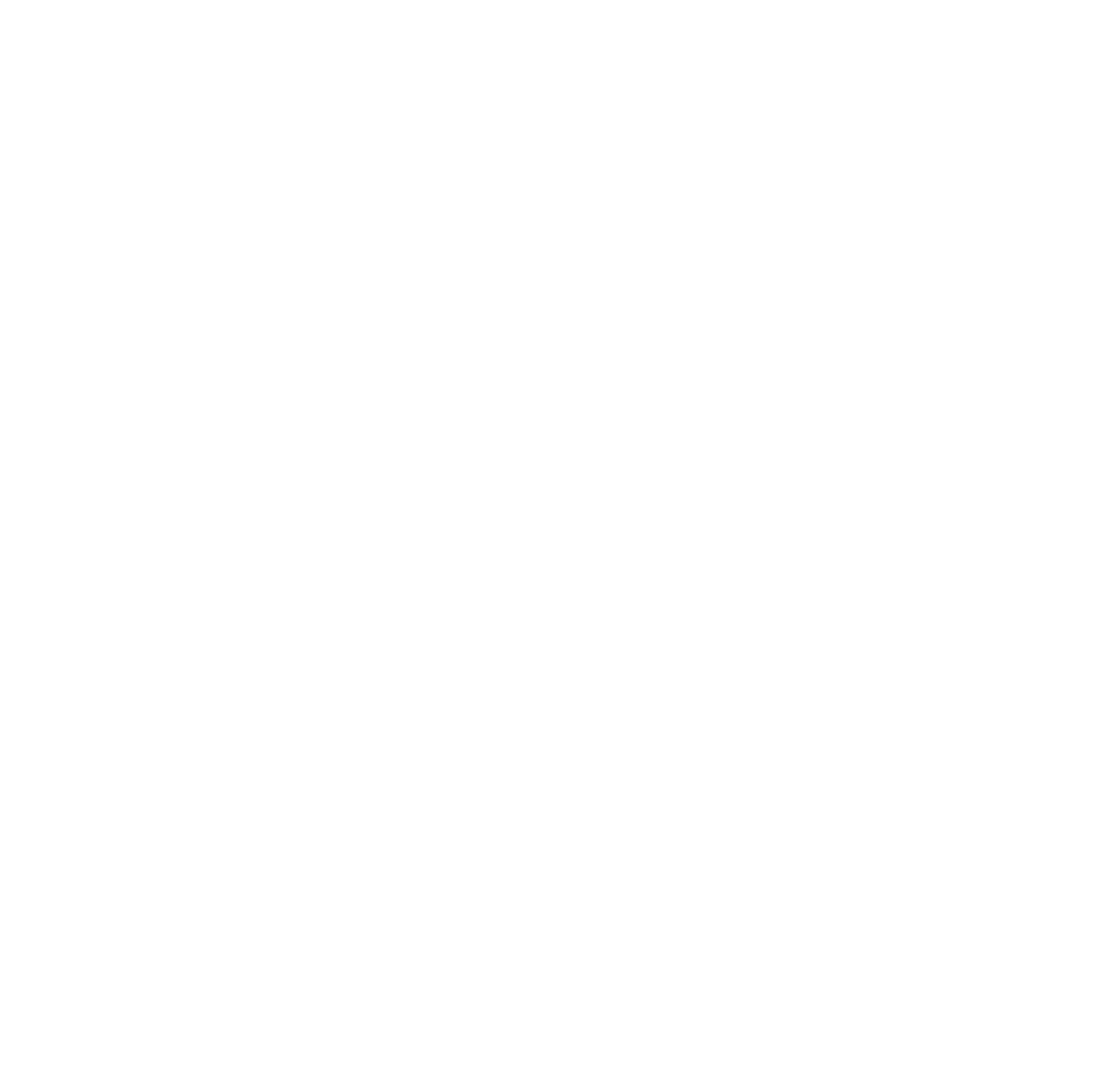

